\title{
Noninvasive mapping of the electrically stimulated mouse brain using photoacoustic microscopy
}

Erich W. Stein, Konstantin Maslov, Lihong V. Wang

Erich W. Stein, Konstantin Maslov, Lihong V. Wang, "Noninvasive mapping of the electrically stimulated mouse brain using photoacoustic microscopy," Proc. SPIE 6856, Photons Plus Ultrasound: Imaging and Sensing 2008: The Ninth Conference on Biomedical Thermoacoustics, Optoacoustics, and Acoustooptics, 68561J (28 February 2008); doi: 10.1117/12.769508 


\title{
Noninvasive mapping of the electrically stimulated mouse brain using photoacoustic microscopy
}

\author{
Erich W. Stein, Konstantin Maslov, Lihong V. Wang* \\ Optical Imaging Laboratory, Department of Biomedical Engineering, Washington University in St. \\ Louis, St. Louis, Missouri, USA 63130 \\ Email: estein@biomed.wustl.edu, lhwang@biomed.wustl.edu*
}

\begin{abstract}
Photoacoustic imaging techniques possess high optical contrast with ultrasonic resolution while exceeding imaging depths of pure optical techniques, affording high resolution images deep within scattering biological tissues. In this work, we employ reflection-mode photoacoustic microscopy to non-invasively monitor hemodynamic contrasts and map brain activity. Changes in vascular dynamics of the mouse somatosensory cortex were evoked through electrical stimulation of the hindpaw, resulting in increased photoacoustic intensities spatially correlated with contra-lateral vasculature. Results demonstrate the ability to map brain activation with vascular resolution in three-dimensions, as well as monitor single-vessel hemodynamics with millisecond temporal resolution. Furthermore, these results implicate the feasibility of photoacoustic microscopy to probe intra-cortical single-vessel hemodynamics and pave the way for more extensive functional brain imaging studies.
\end{abstract}

Keywords: photoacoustic microscopy, functional brain imaging, brain hemodynamics

\section{INTRODUCTION}

Currently, there are no imaging modalities that allow high resolution, noninvasive imaging of direct brain activity (i.e., electrical signals). However, it is well known that local hemodynamic changes in oxygenation, volume, and flow within active brain regions arise from the increased metabolic demands of active brain tissue. Therefore, local hemodynamic changes have been used as a downstream surrogate to map brain activity. ${ }^{1}$ Despite the underlying neuralhemodynamic coupling mechanisms not being well characterized, technologies like functional magnetic resonance imaging (fMRI), laser speckle flowmetry, laser Doppler flowmetry, diffuse optical tomography (DOT), and multispectral reflectance imaging rely on neural-hemodynamic coupling to image brain activity. ${ }^{2-6}$ These techniques, however, can not resolve the brain cortex vasculature of small animals noninvasively; thus, noninvasive functional images (if obtainable) exhibit poor spatial resolution. Therefore, a functional imaging technique affording noninvasive resolution of cortex vessels has the potential to provide researchers with the ability to study dynamic brain function over an animal's lifetime, while eliminating risks associated with routine surgeries to expose the brain vasculature and improve image quality.

Photoacoustic (PA) imaging is a hybrid imaging modality with optical absorption contrast and ultrasonic resolution. ${ }^{7}$ Pulsed laser induced ultrasonic waves (PA waves), which are detected with an ultrasonic receiver, arise from absorbed optical energy distributions within tissues; thus, PA imaging provides contrast that is proportional to optical absorption. Multiwavelength PA imaging can provide functional images based on blood oxygenation and volume since the optical absorption properties of hemoglobin, a dominant endogenous absorber, are oxygen-dependent. ${ }^{8}$ Unlike PA contrast, the resolution of PA imaging systems is determined by the ultrasonic detector properties - central frequency, bandwidth, and numerical aperture (NA). Furthermore, since PA wave generation can be induced by diffuse photon absorption and ultrasonic scattering in biological tissues is two orders of magnitude less than that of optical scattering, PA imaging techniques are well suited to image structures below one transport mean free path in tissue $(1 \mathrm{~mm})$. In fact, previous work has shown that photoacoustic tomography (PAT) can resolve structures $5 \mathrm{~cm}$ deep within biological tissues. ${ }^{9}$ Furthermore, PAT has been used to noninvasively image brain vasculature in rats in vivo, as well as to provide noninvasive, high resolution functional maps of brain activity by tracking local changes in cerebral blood volume arising from somatosensory stimulation. ${ }^{10}$ This study demonstrated the feasibility of using PA imaging to map brain activity.

Photons Plus Ultrasound: Imaging and Sensing 2008: The Ninth Conference on Biomedical

Thermoacoustics, Optoacoustics, and Acousto-optics, edited by Alexander A. Oraevsky, Lihong V. Wang,

Proc. of SPIE Vol. 6856, 68561J, (2008) - 1605-7422/08/\$18 - doi: 10.1117/12.769508 
Recently, functional photoacoustic microscopy (fPAM) was introduced as a viable tool for imaging vasculature in vivo. ${ }^{8,11}$ fPAM, which utilizes dark-field illumination, exhibits high transverse resolution (45 $\left.\mu \mathrm{m}\right)$ and axial resolution $(15 \mu \mathrm{m})$ with an imaging depth of $3 \mathrm{~mm}$ in tissue. The oxygen-dependent optical absorption properties of hemoglobin have been exploited in multiwavelength fPAM imaging to map blood oxygenation levels of subcutaneous vasculature networks in vivo. Unlike PAT, which typically uses an unfocused ultrasonic detector and an inverse algorithm to construct images, fPAM uses reflection-mode detection with a spherically focused ultrasonic detector to directly form volumetric images. fPAM, however, has not been applied to image brain vasculature. In this work, we used fPAM to image mouse brain cortex vasculature noninvasively in vivo with a high spatial resolution and contrast to noise ratio (CNR). Furthermore, we noninvasively imaged local changes in cortex blood volume arising from somatosensory stimulation accomplished by electrical stimulation of the hindpaw and obtained real-time hemodynamic profiles within single-vessels. The results of this work implicate the feasibility of fPAM to probe cortex hemodynamics and lay the groundwork for additional functional brain imaging studies.

\section{MATERIALS AND METHODS}

\subsection{Photoacoustic imaging system}

A schematic detailing the fPAM system used in this work is shown in Fig. 1. Photoacoustic excitation was achieved through a tunable pulsed laser system consisting of a tunable dye laser (Cobra, Sirah) pumped by a Nd:YLF laser (INNOSLAB, EdgeWave) with pulse durations of $8 \mathrm{~ns}$ and variable repetition rates up to $5 \mathrm{kHz}$. The dye laser gain medium was Rhodamine 590, also known as Rhodamine 6G, which provides usable output wavelengths from 560 - 580 $\mathrm{nm}$. Dye laser output was coupled to a $1 \mathrm{~mm}$ optical fiber, which was fixed to a precision three dimensional translation stage. Fiber output was sampled by a beam sampler, coupled to an additional optical fiber, and measured by a fast photodiode to provide an intensity reference used to compensate for laser power fluctuations. A conical lens was used to achieve an annular illumination pattern which was then weakly focused into the sample with a condenser. The resulting dark-field illumination pattern reduces optical fluence at the sample surface, partially averages superficial heterogeneities, and reduces superficial paraxial contributions. ${ }^{11}$ Maximum optical fluence was measured to be 12.1 $\mathrm{mJ} / \mathrm{cm}^{2}$, less than the ANSI safety limit for optical energy between 400 and $700 \mathrm{~nm}\left(20 \mathrm{~mJ} / \mathrm{cm}^{2}\right)$. PA waves were detected by an ultrasonic transducer (central frequency: $20 \mathrm{MHz}$; nominal bandwidth: 80\%; NA: 0.75) whose output was amplified $24 \mathrm{~dB}$ by a low-noise, wide-bandwidth $(0.1-500 \mathrm{MHz})$ amplifier (Mini-Circuits). The amplified PA signal was digitized at a $200 \mathrm{MHz}$ sampling rate by a 14 bit data acquisition system (GaGe) and stored on the hard disk of a personal computer. The motor controller, which was synchronized with the internal clock of the data acquisition system, was used to trigger laser emission and data acquisition depending on scan head position (Fig. 1, system components located within the dashed box). For example, if $20 \mu \mathrm{m}$ step sizes in the $x$-direction were desired, the motor controller would trigger laser emission and data acquisition as the scan head traversed each $20 \mu \mathrm{m}$ increment of travel throughout the $x$-direction.

fPAM images were formed by recording time-resolved PA waves for $2.5 \mu$ s at each scan head position, so called A Lines. Based on the sound velocity in soft tissue $(1.54 \mathrm{~mm} / \mu \mathrm{s})$ the recorded time-resolved PA data were converted into a one-dimensional depth-resolved image along the $z$-axis. The scan head was raster scanned throughout $x$ and $y$ to produce a volumetric image of the sample. In this work, two types of images were used: maximum amplitude projection (MAP) images along the $z$ axis and MAP images along the $y$ axis. Furthermore, since this fPAM system used a $20 \mathrm{MHz}$ transducer (the previously characterized system was based on a $50 \mathrm{MHz}$ transducer) ${ }^{8,11}$ transverse and axial resolution was characterized based on the point spread function acquired by imaging a $6 \mu \mathrm{m}$ carbon fiber. Transverse resolution was determined to be $57 \mu \mathrm{m}$ and axial resolution was determined to be $38 \mu \mathrm{m}$. The maximum imaging depth was characterized through the intact skin and skull of a mouse in situ, $3.2 \mathrm{~mm}$, and the abdominal tissue of a rat in situ, 4 $\mathrm{mm}$. 


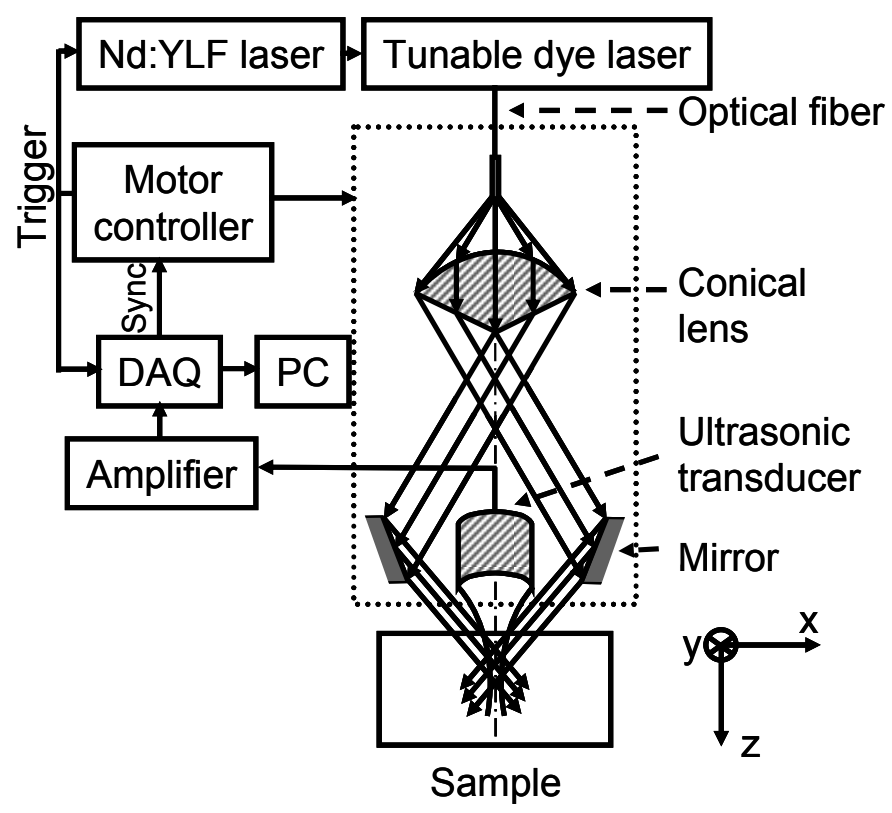

Fig. 1. Schematic of the fPAM system. Components within the dashed box were immobilized on a translation stage and raster scanned along the $x$ and $y$ directions.

\subsection{Animal preparation}

All in vivo procedures were carried out in conformity with the guidelines of the Unites States National Institutes of Health. Furthermore, all in vivo procedures were performed in compliance with the Washington University Animal Study Committee's requirements for the care and use of laboratory animals in research.

Swiss Webster mice (Harlan Sprague Dawley Inc., $\sim 25 \mathrm{~g}$ ) were used. The animal was initially anesthetized using a cocktail $(83 \mathrm{mg} / \mathrm{kg}$ Ketamine and $17 \mathrm{mg} / \mathrm{kg}$ Xylazine) administered by intraperitoneal (IP) injection. Following preparation, the animal was transitioned to vaporized isoflurane $\left(1 \mathrm{~L} / \mathrm{min} \mathrm{O}_{2}, 0.75 \%\right.$ isoflurane) for the duration of all experimental procedures. Depth of anesthesia was monitored by assessing the toe-pinch reflex. Prior to imaging, the hair on the scalp was removed with an over-the-counter depilatory cream. The animal was mounted in a stereotaxic frame and secured with a pair of ear pins and a tooth bar. Body temperature was maintained at $37^{\circ} \mathrm{C}$ with a temperature controlled heating pad. Following the completion of all experimental procedures, the animal was euthanized with a pentobarbital overdose administered by tail vein injection.

After the animal was fixed in the stereotaxic frame, a pair of needle electrodes was inserted under the skin of each hindpaw. Somatosensory stimulation though electrical stimulation of the hindpaw was applied with a current pulse generator at a pulse frequency of $3 \mathrm{~Hz}$, pulse duration of $0.2 \mathrm{~ms}$, and pulse amplitude of $2 \mathrm{~mA}$. In previous reports, these stimulation parameters evoked hemodynamic changes in the vascular network of the rodent somatosensory cortex. ${ }^{6,12}$

\subsection{Functional image construction}

For functional image acquisition, laser wavelength was tuned to $570 \mathrm{~nm}$, a hemoglobin isosbestic point where absorption is independent of oxygenation. As a result increases (or decreases) in hemoglobin absorption at $570 \mathrm{~nm}$ reflect increases (or decreases) in total hemoglobin concentration or blood volume. Functional images $\left(I_{F}\right)$ were constructed by first obtaining a control image $\left(I_{C}\right)$ of the somatosensory cortex, during the acquisition of which no electrical stimulation was applied. Immediately following control image acquisition, a stimulation image $\left(I_{S}\right)$ of the same region was acquired, during the acquisition of which electrical stimulation was applied to the hindpaw. Using equation 1 , the functional image was constructed. 


$$
I_{F}=\frac{I_{S}-I_{C}}{I_{C}}
$$

Thus, the functional images presented in this work represent the fractional difference in PA amplitude with respect to control. Image acquisition time for was approximately $4 \mathrm{~min}$, with total acquisition time for both control and stimulation images being approximately 8 min.

\subsection{Monitoring single-vessel dynamics}

fPAM was also used to track hemodynamic profiles in single somatosensory vessels. First, an image of the somatosensory cortex vasculature was acquired. The scan head was then focused on a single vessel and remained stationary throughout the experiment. Additionally, the laser repetition rate was fixed at $200 \mathrm{~Hz}$ and the wavelength tuned to $570 \mathrm{~nm}$. Hemodynamic profiles were acquired by collecting A Lines at $200 \mathrm{~Hz}$ for $10 \mathrm{sec}$ to establish the signal baseline. Next, while collecting data, 30 seconds of electrical stimulation was applied using the same parameters described above. Finally, stimulation was halted and additional data were collected for $80 \mathrm{sec}$ to allow the response to return to baseline. A hemodynamic profile was obtained by plotting the change (with respect to baseline) of the PA signal originating from within the vessel versus time.

\section{RESULTS AND DISCUSSION}

\subsection{Noninvasive imaging of in vivo brain structure}

Prior to performing functional imaging, the vasculature of the mouse brain cortex was noninvasively imaged in vivo using $570 \mathrm{~nm}$ illumination (Fig. 2). Major vessels in the cortex, as well as the middle cerebral artery and superior sagittal sinus, are clearly imaged. However, a clear view of the olfactory bulbs as well as the cerebellum is obscured by the nasal and intraparietal bones, respectively. Furthermore, the junction between the frontal and parietal bones partially obscures a small region of the cortex vasculature due to increased ultrasonic attenuation associated with increased bone thickness along the suture line (Fig. 2, F/PS).
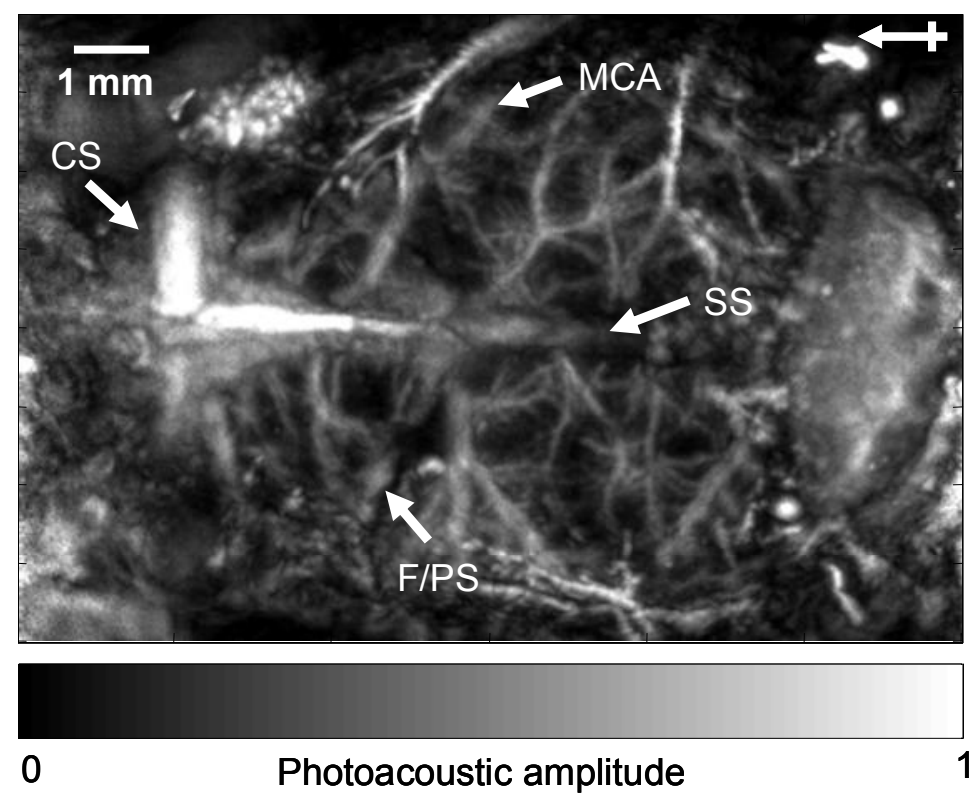

Fig. 2. Noninvasive in vivo fPAM image of mouse cortex vasculature. MAP image projected along the $z$-axis. CS: coronal suture; SS: superior sagittal sinus; MCA: middle cerebral artery; F/PS: frontal-parietal suture. Crossed arrow in the top image points in the rostral direct and is used to indicate image orientation. 
Additionally, the average contrast to noise ratio (CNR) for the cortex vasculature was calculated to be $13 \pm 2 \mathrm{~dB}$. Maximum CNR was calculated to $24 \mathrm{~dB}$; however, this value was observed within the highly vascularized region of the coronal suture (Fig. 2, CS) and was not considered since this work focused on hemodynamics within the cortex.

\subsection{Noninvasive imaging of in vivo brain function}

Local hemodynamic changes in oxygenation, volume, and flow within active brain regions arise from the increased metabolic demands of local neural activity. We used fPAM to noninvasively image changes in blood volume within the right somatosensory cortex evoked by electrical stimulation of the left hindpaw (Fig. 3). As previously stated, laser illumination was tuned to $570 \mathrm{~nm}$, where blood absorption is independent of oxygenation; therefore, changes in absorption result from changes in total hemoglobin concentration or blood volume. Furthermore, it is important to note that the central wavelength of the ultrasonic detector is $75 \mu \mathrm{m}$, which is less than the absorption length of whole blood at $570(\sim 100 \mu \mathrm{m}$, assuming $45 \%$ hematocrit). Therefore, the PA signals acquired were dependent on the blood absorption coefficient.

\section{A}

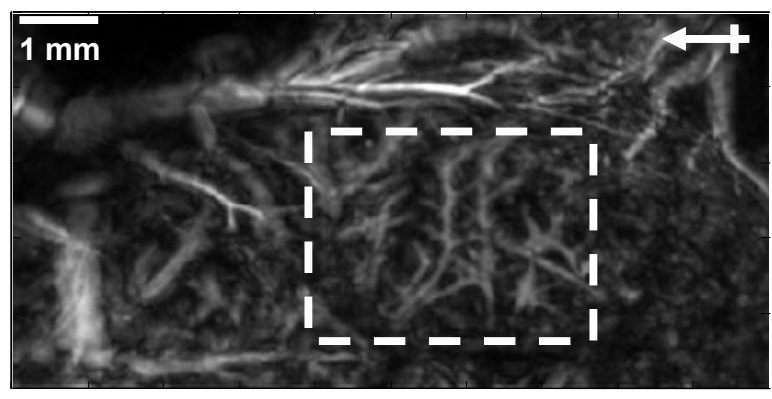

B
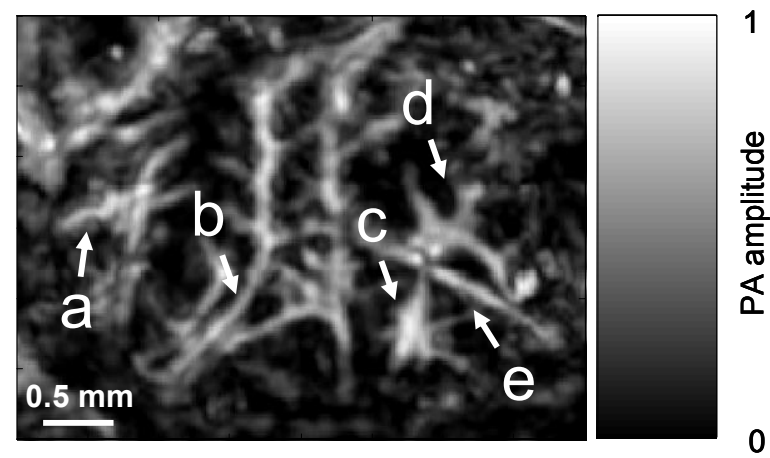

1

$\frac{0}{2}$
$\frac{3}{2}$
$\frac{1}{0}$
$\frac{\pi}{0}$
$\frac{1}{0}$
0
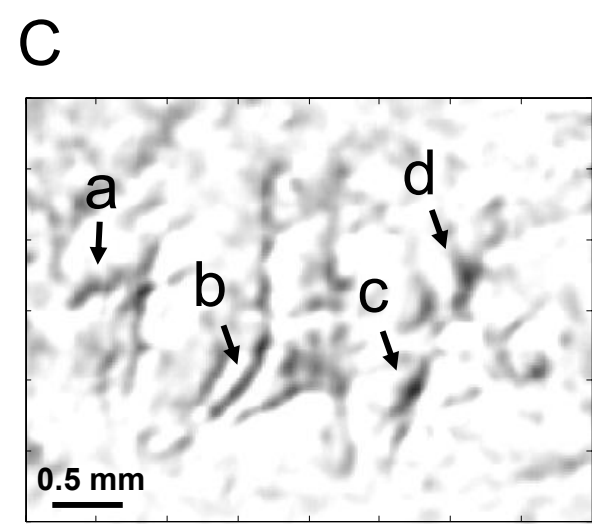

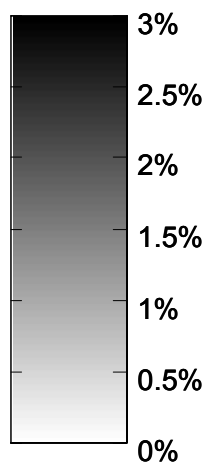

D

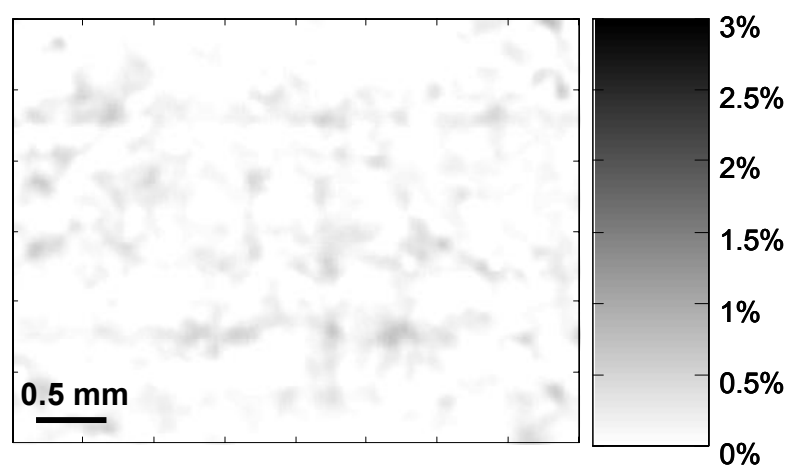

Fig. 3. Noninvasive in vivo fPAM images of mouse cortex vasculature structure and function. A. Image of the right mouse cortex, with dashed box enclosing the somatosensory cortex and crossed arrow pointing in the rostral direction. B. Image of the right somatosensory cortex. C. Functional image depicting percent change in PA signal arising from left hindpaw stimulation. Activation in areas a-d corresponds with vessel structures shown in B. D. Functional control image depicting percent change between images collected without hindpaw stimulation.

Initially, a full view of the right cortex vascular network was obtained (Fig. 3A) and the somatosensory cortex identified (Fig. 3A, dashed box). Fig. 3B represents a high magnification structural image of the right somatosensory cortex vasculature with vessels (Fig. 3B, a-e) clearly seen. A functional image was acquired by sequentially collecting a 
pair of images (one without hindpaw stimulation - the control - and one with hindpaw stimulation) and calculating the fractional difference in PA amplitudes with respect to control. The resulting functional image is shown in (Fig. 3C). The changes in PA amplitude are clearly localized to within the somatosensory cortex vasculature, as indicated by the correspondence of activation areas a-d highlighted in Fig. 3C to vessels a-d highlighted in Fig. 3B. The maximum percent change in PA amplitude was $2.5 \%$. This value is greater than the $\sim 0.5 \%$ change in total hemoglobin concentration observed with diffuse optical tomography ${ }^{6}$ measurement but less that the $\sim 8 \%$ change observed in photoacoustic tomography measurements. ${ }^{10}$ This discrepancy most likely arises from animal-to-animal variations and use of different anesthesia mechanisms, ${ }^{13,14}$ as well as differences in stimulation methods. Additionally, all of the vessels imaged appear to have some degree of activation, an observation arising from continuous stimulation for approximately $4 \mathrm{~min}$. This global activation within the somatosensory region demonstrates that hemodynamic tracking as a downstream surrogate for neural activity imposes physiological constraints on both temporal and spatial resolution due to the delay and spread of the hemodynamic response.

To characterize variation occurring during collection of the control and stimulation images, two images were collected using the same acquisition parameters; however during the collections of these images no electrical stimulation was applied to the hindpaw. The resulting functional control image is displayed in Fig. 3D and indicates no significant change, suggesting that the changes observed in Fig. 3C are due to neuronal activation elicited from hindpaw stimulation. Additionally, we point out that no significant changes in regions outside of the somatosensory vasculature were observed when the hindpaw was electrically stimulation. This observation seems contradictory given previous work that showed significant hemodynamic changes occur in the somatosensory cortex microvasculature following somatosensory stimulation. ${ }^{15}$ The microvasculature is not resolvable with the current PPAM system; thus, the microvasculature contribution would be volume averaged, producing low, undetectable frequencies.

\subsection{Noninvasive tracking of single-vessel hemodynamics}

fPAM was also used to noninvasively monitor hemodynamic changes within a single somatosensory cortex vessel following hindpaw stimulation. First an image of the right cortex was acquired and a single vessel within the somatosensory cortex identified. With the scan head stationary, A Lines were collected at $200 \mathrm{~Hz}$ for a total of 120 seconds. Ten seconds after A Line collection was initiated, 30 seconds of hindpaw stimulation was applied. The raw data depicting the integral of the FWMH of the PA signal originating from within the vessel throughout the experiment was smoothed using the moving average method (Fig. 4A). Given that an A Line is a one-dimensional depth-resolved image, the temporal integrated signal from the tissue above the vessels was also examined (Fig. 4B). As expected, these results show a stable baseline (within noise measurements) during the first 10 seconds of data collection, which were prior to the application of hindpaw stimulation. After the onset to hindpaw simulation, the relative change in the PA signal occurring within the vessel increased at an approximate rate of $0.15 \%$ per second, with a total response time of approximately 12 seconds. The PA signal increase results from an increase in blood supplied to the somatosensory cortex to meet the elevated metabolic demands of active somatosensory neurons. The response stabilizes, then after electrical stimulation is ceased the response returns to baseline. The half-time of the response decay was approximately $11 \mathrm{sec}$. We note that the overall profile acquired, as well as the response and decay times, from within a single somatosensory cortex vessel during hindpaw stimulation was similar to those previously reported using similar stimulation parameters. ${ }^{6}$ The maximum percent change observed was $\sim 2.5 \%$, a value similar to those reported in the previous section. Additionally, the relative changes in the PA signal arising from the tissue above the vessel showed no significant change during hindpaw stimulation, indicating that the hemodynamic response was confined to the vasculature, as expected. 

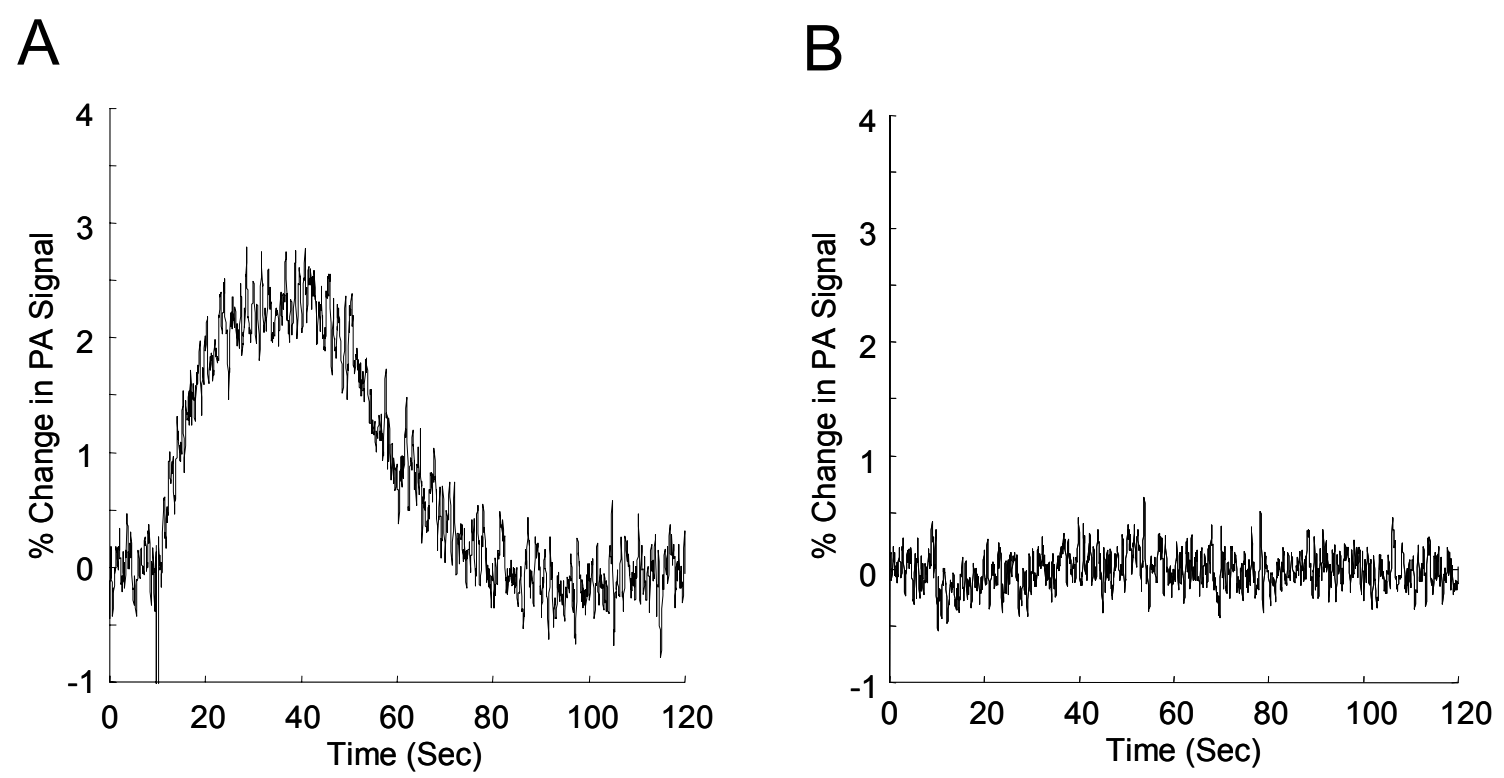

Fig. 4. Noninvasive in vivo fPAM tracking of single-vessel hemodynamic changes resulting from left hindpaw stimulation. A. Data depicting temporal changes in PA signal associated with a single somatosensory cortex vessel during periodic somatosensory stimulation. B. Data depicting temporal changes in PA signal associated with the tissue above a single somatosensory cortex vessel during periodic somatosensory stimulation.

\section{CONCLUSION}

fPAM was used to noninvasively image the structure and function of the in vivo mouse somatosensory cortex. Somatosensory stimulation was achieved by electrical stimulation of the hindpaw, where increased neuronal activity in the somatosensory cortex elicited local hemodynamic changes. Local changes in the blood volume of somatosensory cortex vessels were imaged. The temporal hemodynamic profile within a single somatosensory vessel was also tracked with kilohertz sampling frequency. These results suggest that fPAM is a viable tool for noninvasively monitoring brain activity using hemodynamics as a downstream surrogate for neural activity.

\section{ACKNOWLEDGEMENTS}

The authors greatly acknowledge the National Institutes of Health grants R01 CA106728 and R01 NS46214 (BRP) for financial support.

\section{REFERENCES}

[1] Nair, D. G., "About being BOLD," Brain Res Brain Res Rev 50(2), 229-243 (2005).

[2] Dunn, A. K.; Bolay, H.; Moskowitz, M. A.; Boas, D. A., "Dynamic imaging of cerebral blood flow using laser speckle," J Cereb Blood Flow Metab 21(3), 195-201 (2001).

[3] Dunn, A. K.; Devor, A.; Bolay, H.; Andermann, M. L.; Moskowitz, M. A.; Dale, A. M.; Boas, D. A., "Simultaneous imaging of total cerebral hemoglobin concentration, oxygenation, and blood flow during functional activation," Optics Letters 28(1), 28-30 (2003).

[4] Grinvald, A.; Frostig, R. D.; Lieke, E.; Hildesheim, R., "Optical imaging of neuronal activity," Physiol Rev 68(4), 1285-366 (1988). 
[5] Logothetis, N. K.; Pauls, J.; Augath, M.; Trinath, T.; Oeltermann, A., "Neurophysiological investigation of the basis of the fMRI signal," Nature 412(6843), 150-157 (2001).

[6] Culver, J. P.; Siegel, A. M.; Franceschini, M. A.; Mandeville, J. B.; Boas, D. A., "Evidence that cerebral blood volume can provide brain activation maps with better spatial resolution than deoxygenated hemoglobin," Neuroimage 27(4), 947-959 (2005).

[7] Xu, M. H.; Wang, L. H. V., "Photoacoustic imaging in biomedicine," Review of Scientific Instruments 77(4), (2006).

[8] Zhang, H. F.; Maslov, K.; Stoica, G.; Wang, L. V., "Functional photoacoustic microscopy for high-resolution and noninvasive in vivo imaging," Nat Biotechnol 24(7), 848-851 (2006).

[9] Ku, G.; Wang, L. H. V., "Deeply penetrating photoacoustic tomography in biological tissues enhanced with an optical contrast agent," Optics Letters 30(5), 507-509 (2005).

[10] Wang, X. D.; Pang, Y. J.; Ku, G.; Xie, X. Y.; Stoica, G.; Wang, L. H. V., "Noninvasive laser-induced photoacoustic tomography for structural and functional in vivo imaging of the brain," Nature Biotechnology 21(7), 803-806 (2003).

[11] Maslov, K.; Stoica, G.; Wang, L. V. H., "In vivo dark-field reflection-mode photoacoustic microscopy," Optics Letters 30(6), 625-627 (2005).

[12] Nair, G.; Duong, T. Q., "Echo-planar BOLD fMRI of mice on a narrow-bore 9.4 T magnet," Magnetic Resonance in Medicine 52(2), 430-434 (2004).

[13] Yang, J.; Shen, J.,"Increased oxygen consumption in the somatosensory cortex of alpha-chloralose anesthetized rats during forepaw stimulation determined using MRS at 11.7 Tesla," Neuroimage 32(3), 1317-1325 (2006).

[14] Martin, C.; Martindale, J.; Berwick, J.; Mayhew, J., "Investigating neural-hemodynamic coupling and the hemodynamic response function in the awake rat," Neuroimage 32 (1), 33-48 (2006).

[15] Matsuura, T.; Fujita, H.; Seki, C.; Kashikura, K.; Kanno, I., "Hemodynamics evoked by microelectrical direct stimulation in rat somatosensory cortex," Comparative Biochemistry and Physiology a-Molecular and Integrative Physiology1 24 (1), 47-52 (1999). 